\title{
O USO DA HISTÓRIA DA MATEMÁTICA E DOS OBJETOS DE APRENDIZAGEM COMO FERRAMENTAS PEDAGÓGICAS NA FORMAÇÃO DE PROFESSORES DE MATEMÁTICA
}

\section{THE USE OF HISTORY OF MATHEMATICS AND LEARNING OBJECTS AS PEDAGOGICAL TOOLS IN THE TRAINING OF MATHEMATICS TEACHERS}

\author{
Gisele Pereira Oliveira ${ }^{1}$ \\ Universidade Estadual do Ceará
}

\begin{abstract}
Resumo
Estamos inseridos na era da informação e comunicação, período de grandes transformações educacionais e exigências no currículo brasileiro, tais como, a Base Nacional Comum Curricular (BNCC), em especial, no que diz respeito ao uso de recursos didáticos e tecnologias da informação e comunicação e, com isso, esse trabalho, busca apresentar um recorte de uma pesquisa de Mestrado em ensino de Ciências e Matemática e o potencial existente no uso da História da Matemática (HM) e dos Objetos de Aprendizagem (OAs) como ferramentas pedagógicas na formação de professores de Matemática, em que, mediante a metodologia de pesquisa Engenharia Didática (ED), conseguimos durante o percurso realizar as fases de Análise Preliminar, Concepção e Análise a priori das situações didáticas, Experimentação e Análise a Posteriore e Validação, em que foi possível, em seguimento a essas ações, Conhecer a percepção de professores de Matemática sobre o uso pedagógico da $\mathrm{HM}$ e dos OAs como ferramentas facilitadoras do ensino de Matemática. E, assim, mediante aos dados coletados em um curso de extensão universitária para docentes de Matemática, em período inicial ou continuado de suas práxis, a visualização do uso da HM e dos OAs como ferramentas educacionais facilitadoras de aprendizagem, ainda é muito discreto e, ademais, a execução de atividades, que proponham a professores de Matemática um planejamento eficaz e eficiente de ações, atrelando os recursos e metodologias, afim de potencializar o ensino de Matemática e elevar os saberes do conhecimento matemático dos alunos. E, dessa maneira, concluímos neste estudo, a respeito da importância do uso de ferramentas pedagógicas auxiliadoras e facilitadoras da explanação, representação e experimentação de conceitos matemáticos.
\end{abstract}

Palavras-chave: História da Matemática; Objetos de Aprendizagem; Formação de Professores de Matemática; Ensino de Matemática.

${ }^{1}$ giseleufc@gmail.com

Número Especial - IV Seminário Cearense de História da Matemática

Boletim Cearense de Educação e História da Matemática - Volume 07, Número 20, 126 - 138 (2020)

DOI: $10.30938 /$ bocehm.v7i20.2860 


\begin{abstract}
We are inserted in the information and communication era, a period of great educational transformations and demands in the Brazilian curriculum, such as the National Common Curricular Base (BNCC), especially with regard to the use of didactic resources and information and communication technologies. And, with this, this work seeks to present an excerpt of a Master's research in Science and Mathematics teaching and the potential existing in the use of the History of Mathematics (HM) and Learning Objects (OAs) as pedagogical tools in the formation of Mathematics teachers, in which, through the Didactic Engineering (ED) research methodology, we managed to carry out the stages of Preliminary Analysis, Design and Analysis a priori of didactic situations, Experimentation and Analysis to Posteriore and Validation, in which it was possible , following these actions, To know the perception of Mathematics teachers about the pedagogical use of HM and OAs as tools that facilitate the teaching of mathematics. And so, through the data collected in a university extension course for mathematics teachers, in an initial or continued period of their praxis, the visualization of the use of $\mathrm{HM}$ and OAs as educational tools that facilitate learning is still very discreet and, in addition, the execution of activities that propose to Mathematics teachers an effective and efficient planning of actions, harnessing resources and methodologies, in order to enhance the teaching of Mathematics and raise the knowledge of students' mathematical knowledge. And, in this way, we conclude in this study, regarding the importance of using pedagogical tools that help and facilitate the explanation, representation and experimentation of mathematical concepts.
\end{abstract}

Keywords: History of mathematics; Learning Objects; Mathematics Teacher Training; Mathematics teaching.

\title{
Introdução
}

Estamos vivendo um período de grandes transformações educacionais, em que, as exigências estabelecidas nos documentos oficiais de nosso país, orientadores da educação básica brasileira, passam a exigir formações dos profissionais que fazem parte do cenário escolar e acadêmico.

Essas determinações estão previstas em documentos como a Base Nacional Comum Curricular (BNCC) e os Parâmetros Curriculares Nacionais (PCNs), que são norteadores da educação brasileira e que auxiliam na estruturação do currículo e na organização e planejamento das ações docentes.

$\mathrm{E}$, desse modo, tendo como orientação fundamental a BNCC, documento previsto em lei e integralizado ao currículo da educação básica, conseguimos identificar nesta, a estruturação das áreas do conhecimento em, Linguagens e suas tecnologias, Ciências da Natureza e suas tecnologias, Ciências Humanas e suas tecnologias e Matemática e suas tecnologias; tendo como referência principal, o uso de Tecnologias 
Digitais da Informação e Comunicação (TDIC), já anteriormente, estabelecido nos PCNs como recurso significativo nas práticas docentes.

E, nesse sentido, conseguimos verificar à importância do ensino dos saberes do conhecimento, mediante a inserção de recursos educacionais digitais facilitadores de aprendizagem, buscando continuamente contribuir com o ambiente escolar e acadêmico e, proporcionar um desenvolvimento satisfatório do ensino e aprendizagem destes saberes.

Dessa maneira, podemos destacar, mediante a observação das exigências destes documentos, que o uso das TDIC como ferramentas pedagógicas no ensino das variadas áreas dos saberes do conhecimento tem sido de grande importância, pois como estamos vivendo na era da informação e comunicação, momento em que temos acesso a informação e comunicação com muita facilidade e em rápida velocidade, passa a ser pontual a inserção destes recursos nas práticas educacionais cotidianas.

E, nessa direção, podemos destacar ainda, uma disciplina, citada por muitos discentes, como "difícil e/ou chata", que é a Matemática, uma ciência que ainda apresenta baixos índices nas avaliações internas e externas, tendo como justificativa, diversos fatores, mas que podemos destacar como de grande evidência, a falta de capacitação de professores de Matemática rumo a busca de saberes do conhecimento científico, pedagógico, tecnológico e metodológico, isto é, muitos docentes ainda não possuem a formação, inicial ou continuada, adequada ao uso de recursos educacionais, metodologias de ensino e um planejamento eficaz.

Com isso, percebemos a falta de interesse de muitos alunos por está ciência e os baixos resultados nas avaliações, nos fazendo refletir, segundo os PCNs, que a Matemática, exige o uso de ferramentas pedágogicas e/ou tecnológicas e/ou metodológicas, para o alcançe de saberes do conhecimento científico.

E, nessa compreensão, podemos destacar ademais, como ferramentas favoráveis ao ensino e aprendizagem Matemática, o uso da História da Matemática (HM), que possibilita mediante ao estudo de fatos matemáticos históricos, uma compreensão significativa de assuntos desta, viabilizando um desenvolvimento cognitivo de saberes dos alunos.

E, além disso, podemos pontuar ainda, o uso de Objetos de Aprendizagem (OAs), que são recursos educacionais digitais, disponibilizados na internet, sendo estes, 
O uso da História da Matemática e dos Objetos de Aprendizagem como ferramentas pedagógicas na formação de professores de Matemática

Gisele Pereira Oliveira

idealizados e construídos, com fins educacionais e com pequenas durações, para uso em poucas aulas e para ser objetivo, segundo os propósitos educacionais; tendo uma interface inovadora e atrativa, possibilitando ao aluno a desmitificação de que a Matemática é uma ciência de difícil compreensão, mas que pode ser agradável o seu estudo.

E, também, podemos ainda, estabelecer um paralelo da HM com os OAs como ferramentas facilitadoras do ensino e aprendizagem Matemática, podendo pautar, também, a explanação dos conceitos matemáticos, segundo uma fundamentação da HM, sendo está, ferramenta esclarecedora de assuntos, anteriormente visualizados como de difícil compreensão.

E, em continuidade, podemos além do mais, fazer uso de OAs como ferramentas auxiliadoras na explicação e experimentação de conceitos matemáticos, sendo estes, a partir de sua interface, recurso potencializador de conhecimentos e promotor de um ensino pontual e uma aprendizagem significativa.

$\mathrm{E}$, nesse contexto, vislumbramos o quanto neste processo de ensino e aprendizagem de conceitos matemáticos, atrelados á uma explanação e experimentação de conceitos, segundo a HM e com o uso de OAs, se faz necessário a formação de professores de Matemática sobre o uso destas ferramentas pedagógicas, permitindo através de formações iniciais e continuadas à possibilidade de impulsionar às práticas no ensino de Matemática e, com isso, contribuir com a melhoria das avaliações internas e externas.

E, mediante a essas necessidades e sabendo do papel do meio acadêmico de oferecer o tripé, ensino, pesquisa e extensão, destacamos e percebemos a importância da oferta de formações docentes em caráter de extensão universitária, promovendo a partir destas, a oportunidade de capacitações, iniciais ou continuadas, sobre recursos educacionais, metodologias de ensino e/ou pesquisa e práticas inovadoras.

E, portanto, visualizamos a possível contribuição ao cenário educacional matemático, ao oferecer um curso de extensão universitária, que permitisse a participação de professores de Matemática, em períodos iniciais ou continuados de sua práxis docentes, sendo ofertado, a experiência significativa de "aprender a aprender" o exercício da carreira docente, conforme o uso de TDIC, OAs, HM e metodologias facilitadoras da aprendizagem de conceitos matemáticos. 
E, dando seguimento, realizamos durante uma pesquisa de Mestrado em ensino de Ciências e Matemática, segundo a metodologia Engenharia Didática (ED), a realização de quatro fases, que colaboraram com a formação de professores de Matemática.

E, sendo assim, conforme a ED, realizamos no primeiro momento, a fase, denominada a partir desta metodologia, como Análise Preliminar, fazendo um levantamento bibliográfico de trabalhos correspondentes a temática trabalhada na plataforma Banco de Teses e Dissertações da Coordenação de Aperfeiçoamento de Pessoal de Nível Superior (CAPES) e em programas de cursos, matrizes curriculares e ementários de licenciaturas em Matemática de universidades públicas cearenses, tais como, Universidade Federal do Ceará (UFC), Universidade Estadual do Ceará (UECE), Universidade Estadual Vale do Acaraú (UVA), Universidade Reginal do Cariri (URCA), Universidade da Integração Internacional da Lusofonia AfroBrasileira (UNILAB) e Instituto Federal de Educação, Ciência e Tecnologia do Ceará (IFCE).

No segundo momento, realizamos a fase nomeada por Concepção e Análise a priori das situações didáticas, em que, estabelecemos neste período as variáveis de investigação, a pergunta diretriz, os objetivos, geral e específico, preparamos os materiais utilizados no curso de extensão universitária, que descreveremos na fase seguinte, sendo produzidos ainda, os questionários, inicial e final, para coleta de dados da pesquisa, roteiros de produção de OAs, tendo no mais, a idealização, planejamento e construção de um SITE, por meio da ferramenta gratuíta Google Site, em que, posteriormente, disponibilizamos o relato de experiência, os materiais produzidos e demais recursos usados durante as formações, buscando por meio deste, colaborar com o desenvolvimento do ensino de Matemática.

No terceiro momento, executamos a fase de Experimentação, em que nesta ocasião realizamos um curso de extensão universitária sobre "o uso pedagógico de OAs no ensino de Matemática", sendo apresentado recursos educacionais de caráter concreto e digital, metodologias de ensino e pesquisa, a importância de um planejamento eficiente e eficaz, que atrela-se o uso de ferramentas pedagógicas e metodológicas ao ensino de saberes do conhecimento matemático.

E, ainda sobre a fase anterior, podemos destacar, que dentre as diversas experiências vivenciadas neste momento, produzimos alguns OAs e tivemos professores 
de Matemática, que optaram conforme suas necessidades docente, por produzir um OA que contemplasse a HM, tendo a oportunidade nesse período, de construir um roteiro de produção de OAs e produzir-lo na plataforma ANIMAKER, que é um ambiente de produção de recursos educacionais digitais.

Na quarta fase, Análise a Posteriore e Validação, analisamos os dados coletados na Experimentação, com o interesse de investigar a percepção dos professores de Matemática, em período inicial ou continuado, sobre suas formações docentes, as fragilidades da ausência desta em relação a diversos assuntos e os potenciais desenvolvimentos de aprendizagem adquiridos por conta da participação nesta ocasião.

E, em seguida, ainda no quarto período, segundo a ED, confrontamos, a pergunta diretriz, objetivos e demais variáveis estabelecidas na fase Concepção e Análise a priori das situações didáticas, observando o alcançe destas e permitindo assim, mediante ao uso da ED, a Validação da pesquisa.

E, desse modo, decidimos neste trabalho, apresentar um recorte da pesquisa realizado durante o Mestrado, buscando para está finalidade, responder a pergunta diretriz: "Como a utilização da História da Matemática e o uso de Objetos de Aprendizagem podem contribuir como ferramentas pedagógicas no ensino de Matemática ?".

E, para facilitar a busca por respostas a este questionamento, buscamos Conhecer a percepção de professores de Matemática sobre o uso pedagógico da HM e dos OAs como ferramentas facilitadoras do ensino de Matemática.

\section{A História da Matemática como ferramenta pedagógica na formação de professores de Matemática}

A HM é uma ferramenta de ensino facilitadora da aprendizagem de conceitos, pois permite de acordo com a explanação histórica de fatos relevantes à está Ciência, um aprofundamento e esclarecimento de assuntos matemáticos, tendo em vista, que uma boa fundamentação teórica nos possibilita compreender de forma clara diversos conteúdos. E, segundo Brasil (2018, p. 299):

Cumpre também considerar que, para a aprendizagem de certo conceito ou procedimento, é fundamental haver um contexto significativo para os alunos, não necessariamente do cotidiano, mas também de outras áreas do conhecimento e da própria história da Matemática. No entanto, é necessário 
que eles desenvolvam a capacidade de abstrair o contexto, apreendendo relações e significados, para aplicá-los em outros contextos.

E, além disso, é interessante perceber que a HM é pedagogicamente eficaz e eficiente no exercício da carreira do professor de Matemática, pois, muitas vezes, os alunos não dispertam interesse por Matemática e a caracterizam como uma disciplina "chata ou difícil”, mas que ao ser usada tal ferramenta de contextualização e explanação inicial de conceitos matemáticos, podemos descaracterizar essa impressão e colaborar com uma aprendizagem significativa e um interesse por aprender tal Ciência. E, conforme Brasil (2018, p. 298):

\begin{abstract}
Além dos diferentes recursos didáticos e materiais, como malhas quadriculadas, ábacos, jogos, calculadoras, planilhas eletrônicas e softwares de geometria dinâmica, é importante incluir a história da Matemática como recurso que pode despertar interesse e representar um contexto significativo para aprender e ensinar Matemática. Entretanto, esses recursos e materiais precisam estar integrados a situações que propiciem a reflexão, contribuindo para a sistematização e a formalização dos conceitos matemáticos.
\end{abstract}

E, nesse contexto, conseguimos perceber que o uso da $\mathrm{HM}$ ao ensino de Matemática deve estar atrelado à um planejamento eficaz de ações, em que o docente, verifique o melhor momento de se fazer uso de tal ferramenta, buscando favorecer o ensino e oferecer uma aprendizagem clara de conceitos matemáticos, permitindo ao aluno “aprender a aprender" tal disciplina. E, conseguinte Brasil (1997, p.34):

Em muitas situações, o recurso à História da Matemática pode esclarecer ideias matemáticas que estão sendo construídas pelo aluno, especialmente para dar respostas a alguns "porquês" e, desse modo, contribuir para a constituição de um olhar mais crítico sobre os objetos de conhecimento.

E, dessa maneira, visualizamos a capacidade da HM de responder a certos questionamentos feitos pelos alunos, ao tentarem compreender determinados conceitos matemáticos, pois os mesmos, buscam de forma palpável, justificativas que fundamentem e sustentem os conceitos e assuntos trabalhados na Matemática. Segundo Brasil (1997, p. 32):

\footnotetext{
A História da Matemática mostra que ela foi construída como resposta a perguntas provenientes de diferentes origens e contextos, motivadas por problemas de ordem prática (divisão de terras, cálculo de créditos), por problemas vinculados a outras ciências (Física, Astronomia), bem como por problemas relacionados a investigações internas à própria Matemática.
} 
Desse modo, visualizamos que a HM nos mostra o quanto é importante conhecer suas origens, pois de acordo com as situações vivenciadas no decorrer dos tempos e das necessidades cotidianas e científicas, obtemos o desenvolvimento de conceitos e assuntos da Matemática. E, conforme Brasil (1997, p.34) “A História da Matemática, mediante um processo de transposição didática e juntamente com outros recursos didáticos e metodológicos, pode oferecer uma importante contribuição ao processo de ensino e aprendizagem em Matemática".

Por isso, verificamos que a utilização de recursos educacionais pedagógicos e metodológicos, podem auxiliar o processo de ensino e aprendizagem de Matemática, sendo a HM uma ferramenta capaz de responder a muitos questionamentos estabelecidos pelos alunos durante suas formações escolares e acadêmica, podendo dessa maneira, impulsionar o gosto destes por estudar tal Ciência.

\section{Objetos de Aprendizagem como recursos educacionais digitais na formação de professores de Matemática}

O uso de TDIC no ensino de Matemática, em especial, os OAs, já vem sendo previsto em muitos documentos oficiais brasileiros, norteadores da educação básica e superior, mas que, acabam não sendo usados adequadamente por falta de formações de cunho tecnológico, que oferte ao professor de Matemática, a compreensão correta da usabilidade de tais recursos e o conhecimento que, apenas, a inclusão de tal, não caracteriza a ação como pedagógica, mas sim, o uso pautado em um planejamento eficaz, que ofereça uma utilização correta destes. Conforme Brasil (1997, p. 26):

\footnotetext{
Novas competências demandam novos conhecimentos: o mundo do trabalho requer pessoas preparadas para utilizar diferentes tecnologias e linguagens (que vão além da comunicação oral e escrita), instalando novos ritmos de produção, de assimilação rápida de informações, resolvendo e propondo problemas em equipe.
}

E, nesse contexto, percebemos o quanto é significativo a formação de professores de Matemática, pois mediante a estes momentos, esses profissionais, passam a construir de modo pontual, sua organização mental, de como deve ser a realização correta de práticas de ensino pedagógicas, podendo estes, ter o conhecimento de recursos, tais como OAs, suas localizações gratuítas, tais como, repositórios, nacionais e/ou internacionais, metodologias de ensino e pesquisa e troca de experiências 
exitosas, que possam colaborar com o desenvolvimento cognitivo dos alunos em relação a aprendizagem de Matemática e uma otimização da práxis docente. E, mediante a Castro Filho et al. (2008, p. 2):

\begin{abstract}
Uma das soluções propostas tem sido o desenvolvimento de objetos de aprendizagem (OA), recursos digitais (vídeo, animação, simulação etc) os quais permitem que professores e alunos explorem conceitos específicos em Matemática, ciências, linguagem etc. Embora não haja ainda um consenso sobre sua definição, vários autores concordam que objetos de aprendizagem devam: (1) ser digitais, isto é, possam ser acessados através do computador, preferencialmente pela Internet; (2) ser pequenos, ou seja, possam ser aprendidos e utilizados no tempo de uma ou duas aulas; (3) focalizar em um objetivo de aprendizagem único e (4) serem de fácil utilização.
\end{abstract}

Portanto, o uso de TDIC, OAs, não é simplesmente para se ter mais recursos educacionais digitais para incluir nas aulas de Matemática, mas ferramentas pedagógicas poderosas, em que na era da informação e comunicação, passam a ser mais frequentes no cotidiano dos alunos e, sendo assim, ferramentas tecnológicas de fácil acessibilidade, com interface inovadora e atrativa, acabam estimulando a aprendizagem desta e facilitando o desenvolvimento cognitivo desses. Em conformidade a Wiley apud Castro Filho (2000, p. 2):

Objetos de aprendizagem, doravante denominados de OA, é um termo surgido no início do século XXI para indicar recursos digitais (vídeo, animação, simulação etc.) os quais permitem que professores e alunos explorem conceitos específicos em matemática, ciências, linguagem etc. A ideia de objetos e de reutilização originou-se da programação orientada a objetos.

E, assim, verificamos que a usabilidade de recursos e metodologias, pautados em um planejamento de ações, podem transformar práticas de ensino cotidianas enfadonhas, que na maioria das vezes, não despertam o interesse dos discentes e não geram a aprendizagem desejada, em experiências significativas e impactantes no processo de ensino e aprendizagem Matemática.

\title{
Discussão de Dados
}

Neste trabalho, recorte da pesquisa de Mestrado em ensino de Ciências e Matemática, na fase de Experimentação, conforme a metodologia ED, no período de realização do curso de extensão universitária, com uma oferta de 20 vagas, tendo uma média de participações nos encontros de 16 professores, em período inicial ou continuado, de suas formações, buscamos selecionar situações problemas que 
contemplassem a HM e o uso de OAs, sendo percebidos, a partir da técnica questionário, alguns resultados pertinentes as questões abordadas neste.

E, nessa direção, ao realizarmos a indagação, no questionário inicial, a respeito da formação dos professores participantes, sobre "durante sua formação, os professores trabalharam ou incentivaram o uso de algum recurso didático?”, tendo como opções, sim ou não, em que ao assinalarem sim, deviam destacar quais recursos didáticos foram incentivados, tendo como possibilidades, Material Manipulativo, Jogos, HM, Etnomatemática, Informática Educativa, OAs, Vídeos, Peças Teatrais, Filmes, Softwares e outros, caso não houvesse o item desejado.

$\mathrm{E}$, entre as respostas, tivemos 16 participantes que indicaram a alternativa "sim” e, apenas, um "não". Para os que responderam positivamente, tivemos, com base nas frequências relativas identificadas, $26 \%$ se referiram a material manipulativo, $23 \%$ a jogos matemáticos, $17 \%$ à importância da HM no ensino, 0\% das escolhas para Etnomatemática, Informática Educativa, Peças Teatrais e outros; tendo, ainda, $6 \%$ para OAs, 13\% para Vídeos matemáticos, 4\% indicaram Filmes e 11\% Softwares de Matemáticos.

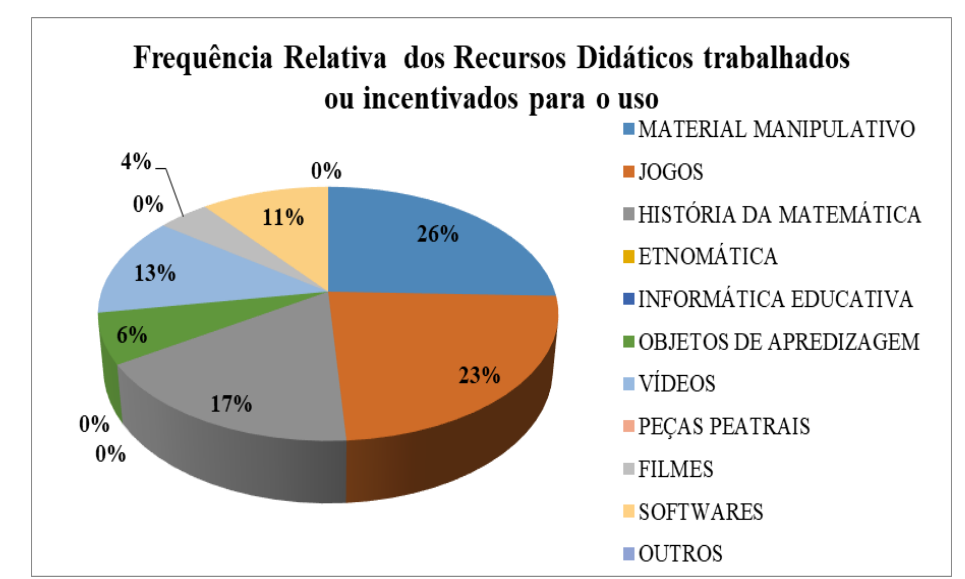

Figura 1 - Gráfico dos recursos didáticos trabalhados ou incentivados para o uso no ensino de Matemática

Fonte: Oliveira (2018, p.79)

E, nesse sentido, observamos o quanto ainda é discreto o uso de recursos didáticos no ensino de Matemática, sendo a $\mathrm{HM}$ e os OAs, ainda sucintos em suas utilizações na práxis docente da disciplina de Matemática, sendo estes, perceptíveis potenciais de ações pedagógicas, geradoras de aprendizagem significativa de conceitos matemáticos. 
E, dando seguimento, aos questionamentos, indagamos ainda, aos que responderam anteriormente como sim, isto é, aos que foram estimulados ao uso de recursos didáticos e, ademais, aos que durante sua formação inicial acadêmica, durante a licenciatura em Matemática, tiveram algum professor que fez uso de OAs em suas aulas, sendo indagados sobre "Em qual(is) disciplina(s) foram utilizados os OAs como ferramentas pedagógicas no ensino de Matemática?”

E, nessa compreensão, tivemos $34 \%$ na de Laboratório de Ensino de Matemática e $11 \%$, respectivamente, em História da Matemática, Álgebra Linear, Prática de Ensino I, Desenho Geométrico, Metodologia do Ensino e Informática Aplicada ao Ensino.

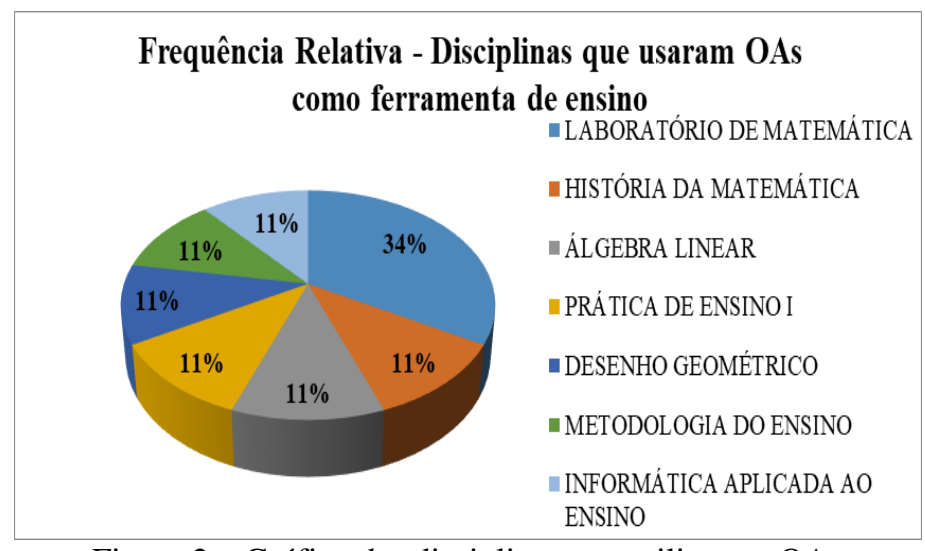

Figura 2 - Gráfico das disciplinas que utilizaram OAs Fonte: Oliveira (2018, p.80).

E, também, tivemos durante o curso de extensão universitária, a produção de roteiros didáticos para construção de OAs pela plataforma ANIMAKER, em dupla de professores, em que, tivemos a ocorrência da construção de um roteiro e OA, que contemplavam a HM, em especial, a Matemática Egípcia, trabalhando o conteúdo de proporção, regra de três simples e equação do primeiro grau, com foco no público do sétimo ano do ensino fundamental final, tendo duração de 2 minutos, sendo objetivo e pontual na abordagem que se desejava tratar, sendo proposto como situação problema neste, a resolução de problemas históricos inseridos no Papiro de Rhind, que é um documento egípcio, de cerca de 1.650 a.C, que detalha problemas de aritmética, geometria e trigonometria básica.

Sendo assim, verificamos as inúmeras contribuições identificadas, mediante ao uso pedagógico da $\mathrm{HM}$ e dos OAs, como ferramentas para o ensino de conceitos 
O uso da História da Matemática e dos Objetos de Aprendizagem como ferramentas pedagógicas na formação de professores de Matemática

Gisele Pereira Oliveira

matemáticos, estimulando dessa maneira os professores de Matemática, em formação inicial ou continuada, da necessidade de um planejamento de ações pontual para a execução no ambiente escolar, oportunizando o desenvolvimento cognitivo dos alunos e contribuindo com os avanços da educação básica e superior.

\section{Considerações Finais}

E, portanto, concluímos a necessidade das formações de professores de Matemática sobre o uso de recursos didáticos e digitais, metodologias de ensino e pesquisa e a sensibilização da realização de um planejamento eficiente de ações, que favoreçam o paralelo entre a usabilidade de recursos e o uso de metodologias as atividades cotidianas no ambiente escolar ou acadêmico, oportunizando mediante a esse processo significativo, práticas pedagógicas de ensino.

E, ainda, destacamos também, o quanto é relevante que as universidades ofereçam a seus licenciandos em Matemática, em período inicial de suas carreiras acadêmicas, um aparato de formações de cunho pedagógico, tecnológico, metodológico e científico, favorecendo suas futuras práticas docentes.

E, nesse contexto e compreensão, chegamos a destacar ademais, que a realização continuada de formações é de suma importância, também, pois capacita continuamente esses professores para evoluírem em suas práxis, conforme o tempo e espaço vivenciados, permitindo uma atualização e experiência qualificadora e inovadora, no que se diz respeito a contextos atuais, possibilitando ao ensino de Matemática um fortalecimento significativo de ações e resultados.

\section{Referências}

BRASIL. Ministério da Educação; Secretaria de Educação Básica. Base Nacional Comum Curricular. Brasília: MEC/ SEB, 2018.600 p.

BRASIL. Secretaria de Educação Fundamental. Parâmetros curriculares nacionais: Matemática / Secretaria de Educação Fundamental. - Brasília: MEC/SEF, 1997. 142p.

CASTRO -FILHO, J.A. Objetos de Aprendizagem e sua utilização no Ensino de Matemática. 2000. Disponível em:

<http://www.pucrs.br/ciencias/viali/tic_literatura/artigos/objetos/Castro_Filho.pdf >. Acesso em: 14 de ago. 2017. 
O uso da História da Matemática e dos Objetos de Aprendizagem como ferramentas pedagógicas na formação de professores de Matemática

Gisele Pereira Oliveira

CASTRO-FILHO, J.A; FREIRE, R. S; FERNANDES, A. C; LEITE; M.A. Quando objetos digitais são efetivamente para aprendizagem: o caso da matemática. In: XIX Simpósio Brasileiro de Informática na Educação - SBIE. Anais [...] Fortaleza, 2008.

OLIVEIRA, G. P. A percepção dos professores de Matemática sobre o uso pedagógico de Objetos de Aprendizagem na formação inicial e continuada. 2018. 156f. Dissertação (Mestrado Profissional em ensino de Ciências e Matemática) Universidade Federal do Ceará - UFC, Fortaleza 2018. 